https://doi.org/10.15407/ujpe65.11.1008

O.I. GERASYMOV, A.YA. SPIVAK

Department of General and Theoretical Physics, Odesa State Environmental University

(15, L'vivs'ka Str., Odesa 65016, Ukraine; e-mail: physics@odeku.edu.ua)

\title{
ON THE WAVE TRANSMISSION \\ IN A GENTLY PERTURBED WEAKLY INHOMOGENEOUS NON-LINEAR FORCE CHAIN
}

\section{Introduction}

The study of the wave transport in complex inhomogeneous media belongs to traditionally actual and complex problems of solid state physics, theory of dynamic systems, and numerical modeling of the wave processes. In the series of papers, the abovementioned problem has been studied in detail [112]. Within the mentioned problems, the non-linear character of the wave transport is superimposed with an inhomogeneity of the medium, where this phenomenon has occurred. The particular attention of the research is paid to low-dimensional media (like, e.g., 1D chains), where the above-mentioned problem can be relatively easily solved numerically or even analytically. It is known after [1-12] that the linear limit of this problem is characterized by quasinormal mode solutions $[6,7,11]$. In the continuous limit, one has a familiar solitonic solutions $[2,4,5]$. In what follows, we focus on the intermittency between non-linear discrete and linear inhomogeneous continuous form equations of the problem and the governing equations. As will be shown below, we have specific wave-modes which approach the familiar solutions in the relevant limits of the interval under study.

(C) O.I. GERASYMOV, A.YA. SPIVAK, 2020

\section{Numerical Solution \\ of the Discrete Non-Linear Equation of Motion}

Consider the system which is constructed, for instance, as a vertical column of $N$ identical grains subject to gravity. Adopt that a current displacement of the $n$-th grain $z_{n}$ satisfies the equation of motion

$\frac{d^{2} z_{n}}{d t^{2}}=\gamma\left\{\left[d-\left(z_{n}-z_{n-1}\right)\right]^{\delta}-\left[d-\left(z_{n+1}-z_{n}\right)\right]^{\delta}\right\}+g$,

where $\gamma=\frac{E \sqrt{d}}{3 m\left(1-\nu^{2}\right)}$ is the force constant, $m$ is the mass of an individual grain, $d$ is the diameter of an unloaded particle, $E$ is the Young elastic modulus, and $\nu$ is Poisson's ratio [13]. The exponent $\delta$ in Eq. (1) could take various values. The Hertzian contacts between beads give rise to $\delta=3 / 2$. In what follows, we will ignore the role of a dissipation.

We have obtained a numerical solution of the general equation (1), by implementing the fourth-order predictor-corrector method of molecular dynamics. In Fig. 1, we plot the results of numerical simulations of the system which is strongly (when differences between the current displacements of nearest particles are much higher than their initial penetration) perturbed by a mechanical impulse which came from

ISSN 2071-0194. Ukr. J. Phys. 2020. Vol. 65, No. 11 
highest outmost particle $(n=1)$. We observe a typical bell-like form of the developed wave with negligible decay and almost conserved dispersion corresponding to the soliton mode.

Despite that the excitation amplitude which is shown in Fig. 1, is relatively small in comparison with the data of the related experimental paper $([5])$, we call this value as a "strong" one. We take into account that when the excitation of a nonlinear system is "weak" (or "gentle"), the resulted governed model equations should be linearized. In other cases (like considered), the excitation is "strong" (in the sense of described above). In the case shown in Fig. 1, the excitation amplitude exceeds the criterion of linearization by 10 times, and is "strong" in this sense, i.e. the system feedback cannot be described by a linear approximation.

\section{Analytic Solution of the Linearized Homogeneous Equation}

Let us introduce a function of the displacement $\varphi_{n}$ which describes a deviation of the $n$-th grain from the initial position:

$\varphi_{n}(t)=z_{n}(t)-\left[(n-1) d-\sum_{k=1}^{n-1} \varepsilon_{k}\right]$

where $\varepsilon_{k}=d-\left(z_{k+1}(0)-z_{k}(0)\right)$ is the overlapping due to the gravity preloading at the point of the $k$-th contact. The substitution of (2) into (1) leads to a nonlinear form of the governing equation in terms of the shift-function $\varphi_{n}$.

The linearized governing equation obtained from Eq. (1) by using a small parameter $\left|\varphi_{n}-\varphi_{n+1}\right| / \varepsilon_{n}<$ 1 can be written in the form

$\frac{d^{2} \varphi_{n}}{d \tau^{2}}=\kappa_{n-1}\left(\varphi_{n-1}-\varphi_{n}\right)-\kappa_{n}\left(\varphi_{n}-\varphi_{n+1}\right)$.

Here, $\tau=t \sqrt{g \delta}\left(\frac{\gamma}{g}\right)^{1 / 2 \delta}, \kappa_{n}=n^{1-1 / \delta}$ can be treated as a new force constant which becomes dependent on the position (on the number of beads) in the column due to the precompression by the gravity. Equation (3) has been obtained, when the simplest equilibrium condition in each contacts is taken in form $\varepsilon_{k}=\left(\frac{g k}{\gamma}\right)^{1 / \delta}$.

Therefore, Eq. (1) turned into the linear homogeneous form given by Eq. (3): the gravity has escaped from the equation, but remains in the force constants which are renormalized. This mapping formally transform the vertical problem into a horizontal one. We have chosen the equilibrium condition in

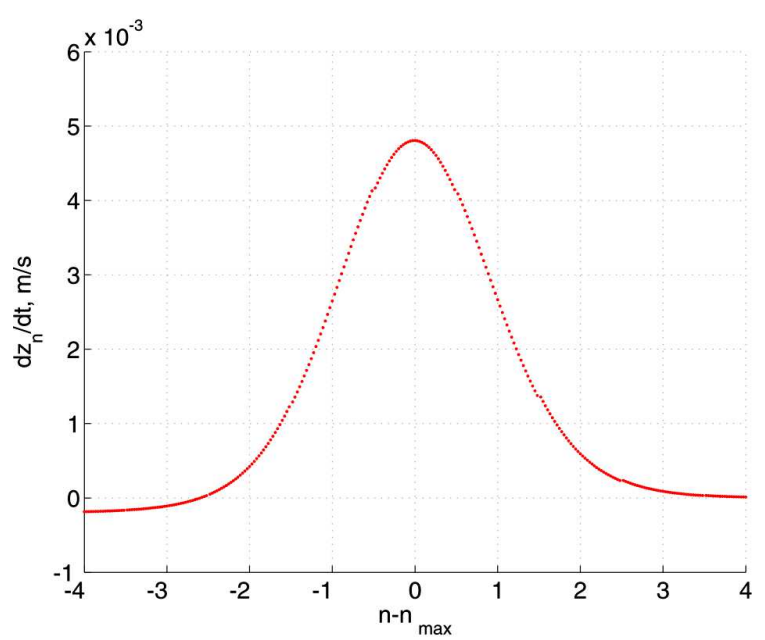

Fig. 1. Velocity of a displacement which is governed by Eq. (1) [Setup: $N=120$ is a number of particles in the Hertz chain $(\delta=3 / 2) ; v_{0}=8.57 \mathrm{~mm} / \mathrm{s}$ is the excitation velocity; $88.4 \div 91.8 \mu$ s is a total time interval during which the mode is developed; $\Delta t=0.1 \mu \mathrm{s}$ is a time step; $g=9.81 \mathrm{~m} / \mathrm{s}^{2}$ is the acceleration of gravity; $d=1 \mathrm{~mm}$ is a particle diameter; $\rho=2426 \mathrm{~kg} / \mathrm{m}^{3}$ is a density; $E=62 \mathrm{GPa}$ is the Young modulus of elasticity; and $\nu$ is Poisson's ratio]

the simplest form of a balance between the gravity and Hertzian forces at the point of each contact. The equations of the type of Eq. (3) belong to the class of functional-differential equations (called differencedifferential equations).

When $\kappa_{n+1} \approx \kappa_{n} \approx \kappa_{n-1} \equiv \tilde{\kappa}$ which can be (approximately) justified for the internal segments $(n \gg 1)$ in a long enough chain, Eq. (3) can be approximately reduced to

$\frac{d^{2} \varphi_{n}}{d T^{2}}=\varphi_{n-1}-2 \varphi_{n}+\varphi_{n+1}$,

where $T=\tau \sqrt{\tilde{\kappa}}$ is a rescaled local time. Although this rescaling (as we saw) is valid only when $n \gg 1$ in the case of homogeneous, Eq. (4) can be considered as a relevant model equation with limited application.

Under the given initial conditions $\left\{\varphi_{p}(0)\right\}$, $\left\{\dot{\varphi}_{p}(0)\right\}$, the solution of Eq. (4) can be written in the form [14]:

$\varphi_{n}(T)=\sum_{p=-\infty}^{\infty} \varphi_{p}(0) J_{2(n-p)}(2 T)+$
$+\sum_{p=-\infty}^{\infty} \dot{\varphi}_{p}(0) \int_{0}^{T} J_{2(n-p)}(2 T) d T$, 


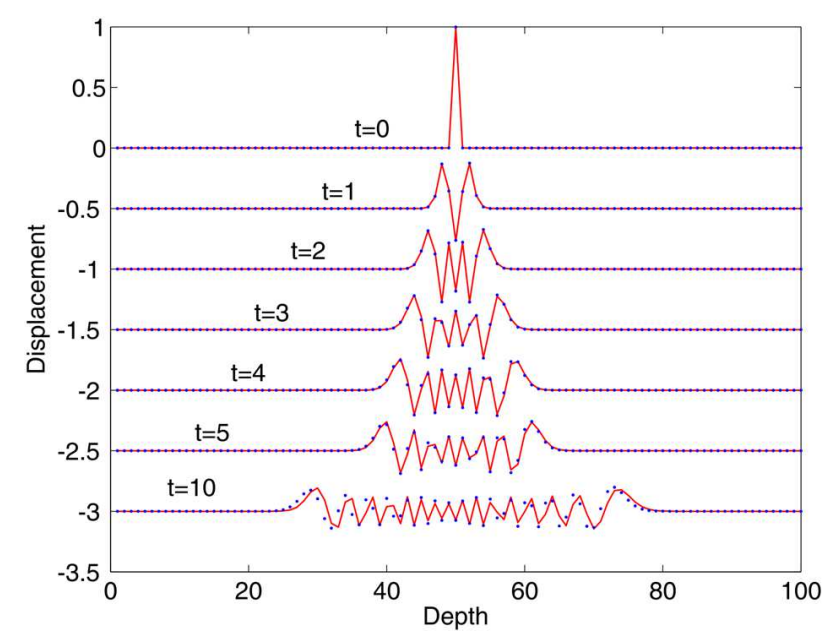

Fig. 2. Displacement $\varphi_{n}(\delta=3 / 2)$ plotted against the depth $n$ at an arbitrarily chosen moment of time: numerical simulation based on Eq. (3) is denoted by points, and theoretical data obtained from Eq. (6) are shown by solid lines (both data correspond to the zeroth initial conditions and a static excitation of the central internal grain, $\varphi_{50}(0)=1$ )

where $J_{2(n-p)}(2 T)$ is the Bessel function of the first kind, and $\dot{\varphi}=\frac{d \varphi}{d \tau}$ is the velocity of a displacement. For the simplest initial condition, when $\varphi_{p}(0)$ (and $\left.\dot{\varphi}_{p}(0)=0\right)$, we have

$\varphi_{n}(T)=\varphi_{p}(0) J_{2(n-p)}(2 T)$.

The main maximum of the Bessel function at the point $n(n \gg 1)$ at the time $t_{1}$ satisfies the condition $\omega t_{1}=2 n$. The velocity of motion of this maximum is $n a \approx \frac{1}{2} \omega a$ and coincides with the sound propagation velocity in a $1 \mathrm{D}$ crystal.

In Fig. 2, the solution of Eq. (6) is plotted in case where the discrete chain has been excited from the middle. Because of the rescaled character of the argument $2 T$, Eq. (6) can be applied in a wide interval of the real times $t$ (see Eq. (8), as well as Eq. (9) and the data presented in Fig. 2). In general, the solutions which are taken in the form of a linear combination of functions like (6) can formally satisfy appropriate initial conditions.

Coming back in Eq. (6) to the dimensional argument, we obtain

$\varphi_{n}(t)=\varphi_{0} J_{2 n}\left(2 \sqrt{n g \delta}\left(\frac{\gamma}{n g}\right)^{1 / 2 \delta} t\right)$.

1010
Passing to the asymptotic limit as $t \rightarrow \infty$, we get from Eq. (7) ([15]):

$\varphi_{n}(t) \sim \frac{1}{\sqrt{\pi \tilde{\tau}}}(n g)^{-1 / 12} \times$

$\times \cos \left(2 \tilde{\tau}(n g)^{1 / 6}-\pi n-\frac{\pi}{4}\right)$,

where $\tilde{\tau}=\sqrt{\frac{3}{2}} \gamma^{1 / 3} t$. The amplitude of $\varphi_{n}(t)$ given by Eq. (8) is $A_{n} \sim(n g)^{-1 / 12}$, and the phase velocity behaves itself as

$v_{p h}=\frac{d n}{d \tilde{\tau}} \sim(n g)^{1 / 6}$.

Similar results (i.e. $A(h) \sim h^{-1 / 12}$ and $v_{p h} \sim h^{1 / 6}$ ) were obtained in [6], in the continuous limit of the governing equation (3). The wave-front of the displacement $v_{p h} \tilde{\tau}$ follows from (9) and satisfies $n \sim \tilde{\tau}^{6 / 5}$. A similar law for the wave front velocity was obtained in [11], where the continuous form of Eq. (3) was used. It is easy to show that both mentioned results in the limit of a homogeneous chain become similar to each other.

Consider now the obtained upper solutions in more details.

The linear combinations of solutions like (6) can satisfy appropriate initial conditions. For instance, the "static" boundary conditions (which can be approached by means of a shift of the outmost particle) look as

$\varphi_{1}(0)=x_{0}, \quad \dot{\varphi}_{1}(0)=0$,

and correspond to a solution in the form of a linear combinations of Bessel functions (see Eq. (5)):

$\varphi_{n}(T)=x_{0}\left[J_{2 n-2}(2 T)+J_{2 n}(2 T)\right]$.

Here, $x_{0}$ is the initial value of a static deformation of the outmost particle.

The "dynamic" boundary conditions (which correspond to the excitation generated by an external impulse) take the form

$\varphi_{1}(0)=0, \quad \dot{\varphi}_{1}(0)=v_{0}$,

and correspond to the solution

$\varphi_{n}(T)=v_{0} \int_{0}^{T}\left[J_{2 n-2}(2 T)+J_{2 n}(2 T)\right] d T$.

ISSN 2071-0194. Ukr. J. Phys. 2020. Vol. 65, No. 11 
In Fig. 3, we give the results of numerical simulation performed with help of Eq. (11) and Eq. (13). One can see a good enough agreement between the analytic solutions given by Eq. (11) and Eq. (13) and the results of direct numerical simulations made with help of Eq. (4).

\section{Analytic Solution \\ of the Inhomogeneous Form of the Governing Equation}

Consider now an analytic approach to the solution of the linear inhomogeneous equation (3) (in what follow, we will follow $[14,16])$.

Consider the probe function for a displacement

$\varphi_{n}(\tau)=X_{n} \cos \omega \tau$

where $X_{n}$ satisfy the differential-difference equation

$\kappa_{n} X_{n+1}-\left(\kappa_{n}+\kappa_{n-1}+\lambda\right) X_{n}+\kappa_{n-1} X_{n-1}=0$.

The solution of Eq. (14) can be obtained in following form $[14,16]$ :

$\varphi_{n}(\tau)=\sum_{p=1}^{N-1} \frac{1}{K_{p}} X_{n}\left(\lambda_{p}\right) X_{k}\left(\lambda_{p}\right) \cos \omega\left(\lambda_{p}\right) \tau$,

where $k$ is the number of a selected particle, which has been initially excited $\left(\varphi_{k}(0)=1\right) ; K_{p}$ is a factor which satisfies the equation

$\sum_{n=1}^{N-1} X_{n}\left(\lambda_{p}\right) X_{n}\left(\lambda_{q}\right)=K_{p} \delta_{p q}$,

where $\delta_{p q}$ is the Kronecker function. Note that, in the case where the outmost particle has been initially moved, $k$ become equal to unity.

For example, in the case of a finite-length chain with $N=2$, Eq. (15) and Eq. (16) give, respectively, the following result:

$\varphi_{1}(\tau)=\cos \tau$.

The above-described algorithm can be repeated without principal difficulties for an appropriate chain length.

In view of the expansion [15]

$\cos (t \sin \phi)=$

$=J_{0}(t)+2 J_{2}(t) \cos (2 \phi)+2 J_{4}(t) \cos (4 \phi)+\ldots$,

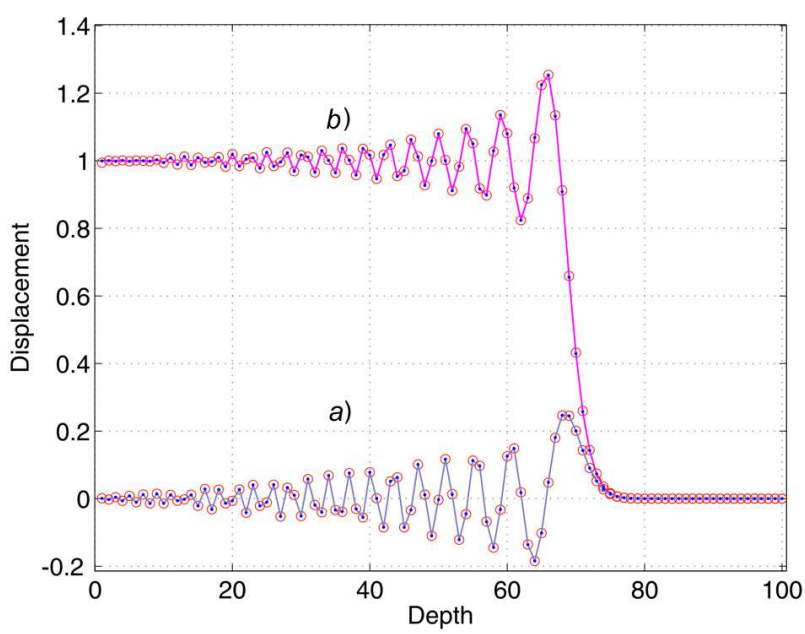

Fig. 3. Displacement $\varphi_{n}(\delta=1)$ plotted vs. the depth $n$ at an arbitrarily chosen moment of time: the resuts of numerical simulation based on Eq. (4) are shown by solid lines, and theoretical data obtained from Eq. (11) and Eq. (13) are presented by circles for static $(a)$ and dynamic $(b)$ excitations, respectively

(where $J_{n}$ is the Bessel function of the first kind, and $\phi$ is a constant phase which can be determined from Eq. (21)), we can present a solution of the inhomogeneous equation (3) in the form

$\varphi_{n}(\tau)=\sum_{j=1}^{\infty} A_{j}(n) J_{2 j-2}\left(2 \sqrt{\kappa_{N}} \tau\right)$,

where $A_{j}(n)$ and $\phi$ [obtained from Eq. (15) and Eq. (18)] are given by

$A_{j}(n)=\sum_{i=1}^{N-1} \frac{X_{n}\left(\lambda_{i}\right) X_{k}\left(\lambda_{i}\right)}{\sum_{m=1}^{N-1}\left[X_{m}\left(\lambda_{i}\right)\right]^{2}} \times$

$\times \begin{cases}1, & i=1, \\ 2 \cos \left[(2 j-2) \phi_{i}\right], & i>1,\end{cases}$

$\phi_{i}=\arcsin \frac{\omega_{i}}{2 \sqrt{\kappa_{N}}}$.

In the simplest case $\left(\kappa_{n}=1\right)$, we have form $\phi_{i}=\frac{\pi}{2} \times$ $\times \frac{2 i-1}{2 N-1}$ (e.g., if $N=3$, we have: $\phi_{1}=18^{\circ}, \phi_{2}=54^{\circ}$, $\left.\phi_{3}=90^{\circ}\right)$.

When $\kappa_{n}=1$, the rigorous solution of Eq. (19) can be simplified (e.g., if $j<N$, we have $A_{n}(n)=$ $\left.=A_{n+1}(n)=1, A_{j \neq n, n+1}(n)=0\right)$ and return to the results given by Eq. (11). 


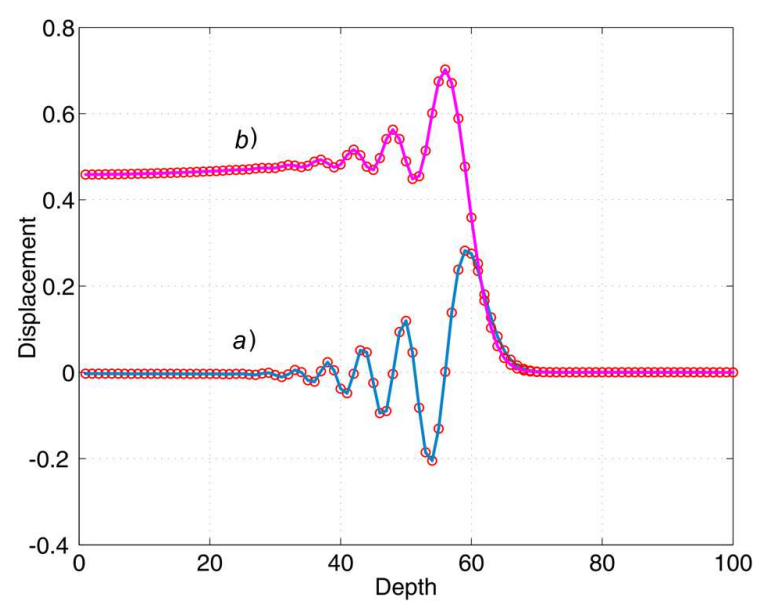

Fig. 4. Displacement $\varphi_{n}(\delta=3 / 2)$ plotted vs. the depth $n$ at an arbitrarily chosen moment of time: numerical evaluation of Eq. (3) is denoted by solid lines, and theoretical data obtained from Eq. (19) are shown by circles for static (a) and dynamic (b) excitations, respectively

Therefore, the rigorous solution of the linearized equations of motion which govern the impulse propagation in an inhomogeneous granular chain shows the oscillation behavior as a function either of the rescaled time $\tau$ or the number of position $n$.

In Fig. 4, we compare the data of numerical simulations made for Eq. (3) and the theoretical results obtained by exactly solving Eq. (19) in the both cases, either static or dynamic excitations.

Figure 5 shows the results of numerical simulations of the general nonlinear equation (1) in comparison with the solution of Eq. (3). We observe a satisfactory correspondence between the solutions of the general nonlinear equation and their linearized inhomogeneous approximation with certain deviations which can be described in terms of Bessel functions by the algorithm given above.

We note that, rigorously speaking, the dynamics of an inhomogeneous weakly nonlinear granular chain could not be described in a single wave approximation, but follows a multimode scenario.

\section{Role of Disorder (after Dyson)}

In spite of the topological ordering of 1D force chains, the concept of disorder, in the sense of Dyson $[17,18]$, can be applied to study the relevant effects in the wave transport through such a system. As was mentioned above, one-dimensional systems are topologi-

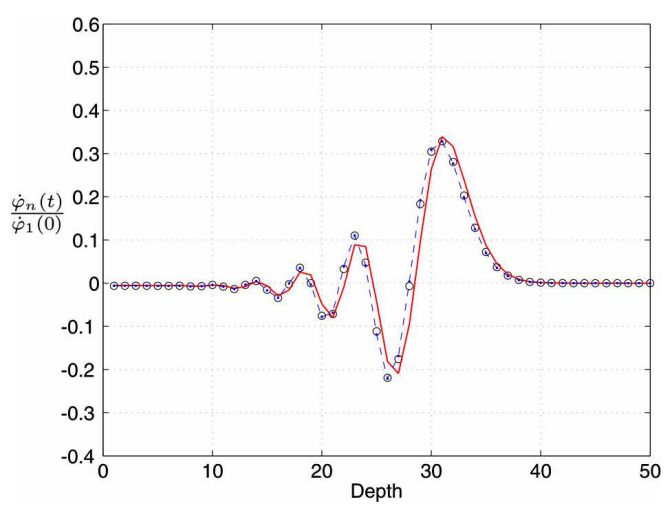

$a$
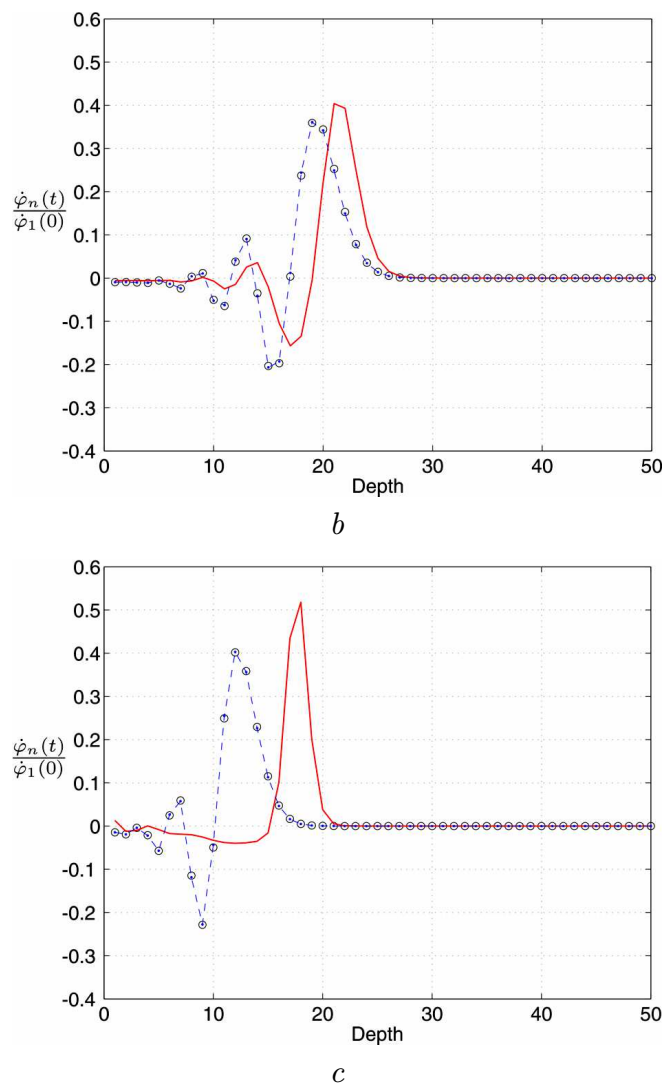

Fig. 5. Relative velocity of a displacement $\dot{\varphi}_{n} / \dot{\varphi}_{1}(0)$ plotted vs. the depth $n$ at an arbitrarily chosen moment of time (taken as $t=100 \mu \mathrm{s}$ with $\delta=3 / 2$ ): numerical simulation of Eq. (1) is denoted by red solid lines; theoretical data obtained from Eq. (19) are shown by circles markers and blue dash lines (at the dynamic initial excitation of the external glass grain $\left.\dot{\varphi}_{1}(0)=0.857 \mathrm{~mm} / \mathrm{s}\right)$. We use the following constitutive constants for glass: $\rho=2426 \mathrm{~kg} / \mathrm{m}^{3}, E=62 \times 10^{9} \mathrm{~Pa}$, $\nu=0.2, d=1 \mathrm{~mm}$, and $\gamma=5.3593 \times 10^{14} \mathrm{~m} / \mathrm{s}^{2}$. Gravity: $10 \mathrm{~g}$ on the top, $g$ on the middle, and $0.1 g$ on the bottom (where $\left.g=9.81 \mathrm{~m} / \mathrm{s}^{2}\right)$

ISSN 2071-0194. Ukr. J. Phys. 2020. Vol. 65, No. 11 
cally ordered. In order to study the effects of disorder in such systems, Dyson was proposed an approach in which the meaning of disorder addressed to a force constant $[17,18]$. Along this way, we adopt a Dyson distribution of the force constants $\left\{\kappa_{n}\right\}$ in Eq. (3), in order to estimate (approximately) how this mechanism affects the velocity of a displacement. For those purposes, we introduce the following characteristic function $\overline{\dot{\varphi}}_{m}$ (where the upper line means the averaging after Dyson):

$\overline{\dot{\varphi}}_{m}=\sum_{n=1}^{N} D_{m}\left(\kappa_{n}\right) \dot{\varphi}_{n}(\tau)$,

where
$D_{m}\left(\kappa_{n}\right)=\frac{m^{m}}{(m-1) !}\left(\kappa_{n}\right)^{m} e^{-m \kappa_{n}}$,

is the Dyson distribution function which characterizes the degree of disorder; $m$ is a parameter (we have an ordered state as $m \rightarrow \infty$ and a disordered one as $m \rightarrow 1)$.

Equation (22) has been solved numerically. Relevant data are presented in Fig. 6 and Fig. 7. As one can see, an increase in the Dyson disorder degree leads to a decrease in the averaged (in the Dyson sense) velocity of a displacement (we used a solution of Eq. (3)). When the system tends to an ordered state, the respective value of the velocity of a displacement increases. Therefore, the system show asymptotically the disappearence of the difference between the ordered and disordered states in terms of the averaged (after Dyson) velocity of a displacement.

\section{Continuous Limit}

\section{of the Wave Transport Problem}

The asymptotic scenario of the wave-transport described by Eq. (3) which governs the wave propagation in a Hertzian chain can be approximated in the so-called continuous limit which looks as follows. Let us make transformations from discrete integers to continuous arguments:

$n \rightarrow h$;

which leads, in turn, to

$\varphi_{n} \rightarrow \varphi(h), \quad \varphi_{n+1} \rightarrow \varphi(h+\partial h) ;$

and to

$$
\begin{aligned}
& \kappa_{n} \rightarrow \kappa(h)=h^{1-1 / \delta}, \\
& \kappa_{n+1} \rightarrow \kappa(h+\partial h)=(h+\partial h)^{1-1 / \delta} .
\end{aligned}
$$

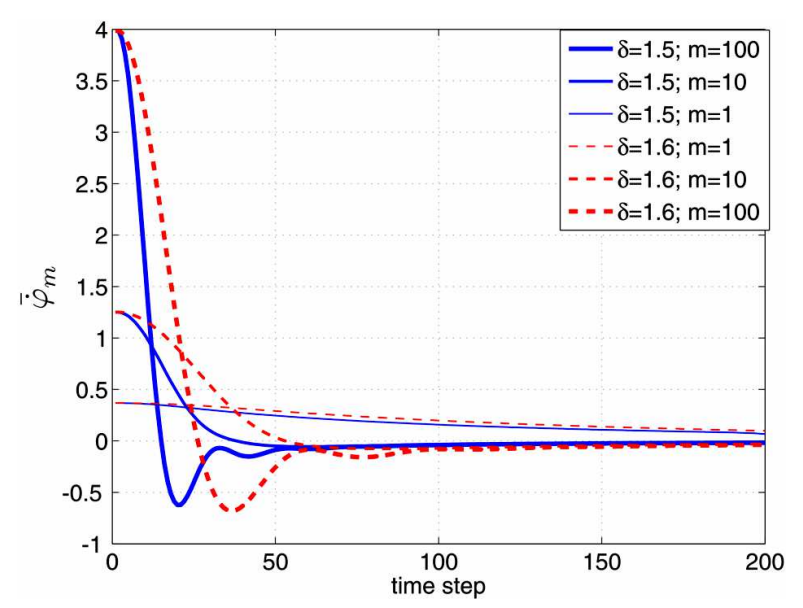

Fig. 6. Behavior of the characteristic function given by Eq. (22) which describes the influence of the Dyson disorder on the transport properties of a Hertzian chain (drosseling-like effect manipulated by the Dyson disorder)

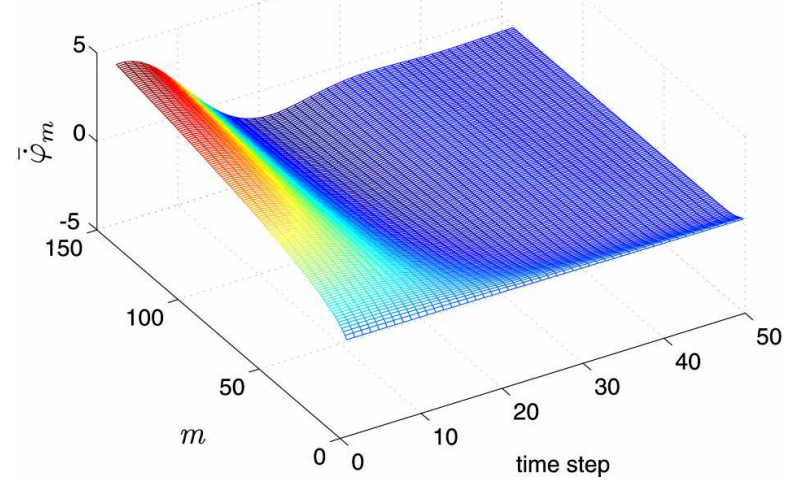

Fig. 7. 3D display of the influence of the Dyson disorder on the wave-transport in a Hertzian chain $(\delta=3 / 2)$

Using Eqs. (24)-(26) and expanding all these approximations into power-series, in the simplest case (when we neglect all derivatives whose order is higher than 2), we obtain

$\frac{\partial^{2} \varphi}{\partial \tau^{2}}=\frac{\partial}{\partial h}\left(h^{\xi} \frac{\partial \varphi}{\partial h}\right)$

where $\xi=1-1 / \delta$. Equations like Eq. (27) are linear and can be solved analytically.

For instance, the simplest solutions which depend from one of the variables ( $h$ or $\tau$ ) only take the form

$\varphi(\tau)=A \tau+B, \quad \varphi(h)=C h^{1 / \delta}+D$,

where $A, B, C, D$ are constants. 


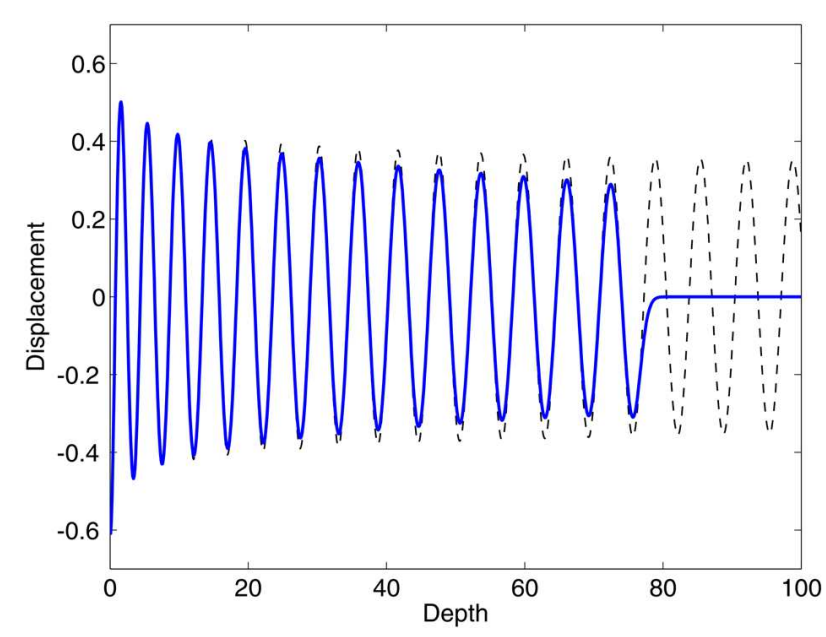

Fig. 8. Displacement $\varphi$ versus the depth $h$ obtained by: numerical solutions of Eq. (27) with periodic boundary condition (denoted by the blue solid line), theoretically with the help of the single mode in Eq. (29) (denoted by black dashed line), where $\delta=3 / 2$

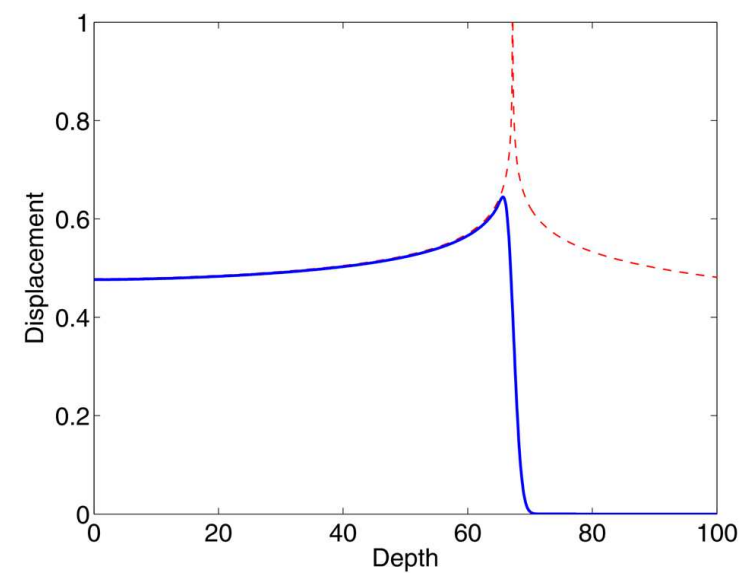

Fig. 9. The soliton-like behavior of the displacement obtained as results of: numerical simulations of Eq. (27) under the dynamic excitation (denoted by the blue solid line); rigorous theoretical solution given by Eq. (35) (denoted by the red dash line), where $\delta=3 / 2$

Equation (27) has also a solution in the form of a quasinormal mode [6]

$\varphi(h, \tau)=\int_{0}^{\infty} u_{\lambda}(h) e^{i \tau \lambda} d \lambda$,

where $u_{\lambda}$ is obtained from an equation of the Bessel type in the following form [6]:

$u_{\lambda}(h)=h^{(\eta-\xi) / 4} H_{\tilde{\nu}}^{(1)}\left(\frac{2 \lambda}{\eta} h^{\eta / 2}\right)$,

1014 where $H_{\tilde{\nu}}^{(1)}$ is a Hankel function of the first order; $\tilde{\nu}=1 /(1+\delta) ; \eta=1+1 / \delta$. Here, $\lambda$ is a parameter which is defined from the relevant Sturm-Liouville problem solution.

Consider linear combinations of expressions from Eq. (29). It follows that the functions given by

$\varphi(h, \tau)=\int_{0}^{\infty} A(\lambda) u_{\lambda}(h) e^{i \tau \lambda} d \lambda$,

satisfy the basic transport equation (27). The modes given by Eq. (31) are attenuated quasinormal modes which can pass the system under the particular amplitude modulation providing plenty of scenarios of the wave dynamics which can be developed in the long-wave limit in the weakly non-homogeneous Hertz chain.

Under the particular selected amplitude modulation law, we got the relevant channeling regime.

For example, when $A=$ const and $\delta=3 / 2$, after the evaluation of the integral in Eq. (31), we obtain

$$
\begin{aligned}
& \Re\{\varphi(h, \tau)\}=\frac{\tilde{A}}{\sqrt{\tau^{2}-\chi^{2}}} \times \\
& \times\left\{\left(\tau+\sqrt{\tau^{2}-\chi^{2}}\right)^{2 / 5}-\left(\tau-\sqrt{\tau^{2}-\chi^{2}}\right)^{2 / 5}\right\},
\end{aligned}
$$

where $\chi=\frac{6}{5} h^{5 / 6}, \tilde{A}=-2 \sin \frac{\pi}{10}\left(\frac{5}{6}\right)^{2 / 5} A$. According to Eq. (32) for $\tau=\frac{6}{5} h^{5 / 6}, \varphi(h, \tau)$ is a limited function. Namely, when $\frac{\tau}{\chi} \rightarrow 1$, one has:

$\lim _{\tau \rightarrow \chi} \Re\{\varphi(h, \tau)\}=\frac{4}{5} \tilde{A} \tau^{-3 / 5}=\frac{4}{5}\left(\frac{5}{6}\right)^{3 / 5} \tilde{A} h^{-1 / 2}$.

Eq. (32) and Eq. (33) show the asymptotic behavior $\left(\sim \tau^{-3 / 5}, \sim h^{-1 / 2}\right)$, and decay much rapidly than solution (35) for $\delta=3 / 2$.

When we select $A \propto \lambda^{-2 / 5}$ and $\delta=3 / 2$, the relevant evaluation in Eq. (31) gives us the expression which has a resonance character, like modes given by Eq. (35). Namely:

$$
\begin{aligned}
& \varphi(h, \tau)=\tilde{B}\left(\tau^{2}-\chi^{2}\right)^{-1 / 10}+ \\
& +\tilde{A} h^{2 / 3} F\left(\frac{1}{2}, \frac{2}{5} ; \frac{7}{5} ; \frac{-\chi^{2}}{\tau^{2}-\chi^{2}}\right)\left(\tau^{2}-\chi^{2}\right)^{-1 / 2}
\end{aligned}
$$

where

$\tilde{A}=-\left(\frac{3}{5}\right)^{2 / 5} \frac{\operatorname{ctg} \frac{2 \pi}{5}}{\Gamma\left(\frac{7}{5}\right)}=-0.2985$

ISSN 2071-0194. Ukr. J. Phys. 2020. Vol. 65, No. 11 
$\tilde{B}=\left(\frac{5}{3}\right)^{2 / 5} \frac{\Gamma\left(\frac{1}{5}\right)}{\Gamma\left(\frac{3}{5}\right)} \operatorname{ctg} \frac{2 \pi}{5}=1.2287$.

Solution given by (35) can be interpreted as a traveling shock-wave perturbation, when the channeling modes can be realized in the case of dispersive impulse input. Therefore, we found that the linear equation (27) can be satisfied by either quasinormal or sharp-peaked (localized) wave solutions.

In Fig. 8, we plot the displacement $\varphi(h, \tau)$ given by Eq. (29) at a fixed moment of time as a function of $h$. As one can see, in the case of periodic perturbation of the system from outmost particles, we observe a monotonic decay of the amplitudes of oscillations.

We found that Eq. (27) us also satisfied by the solutions which has a reminiscence of wave-packet (or soliton-like modes) [19]:

$\varphi(h, \tau)=c_{1}+c_{2}\left|\left(\tau+c_{3}\right)^{2}-\left(\frac{2}{\eta}\right)^{2} h^{\eta}\right|^{-\xi / 2 \eta}$,

where $c_{1}, c_{2}, c_{3}$ are constants. Equation (35) under the condition $\left(\tau+c_{3}\right)^{2}=\left(\frac{2}{\eta}\right)^{2} h^{\eta}$ demonstrates a resonance behavior (see Fig. 9).

Therefore, when we start from a numerical solution of the general nonlinear discrete wave-transport equation, it gives rise to a somewhat like superposition of the normal mode and the wave-packet (soliton-like) of a wave transported through the inhomogeneous Hertzian chain.

The study of the wave-transport at the intermittency between discrete and continuous scenarios shows the existence of preliminary stages of the formation of nonlinear-modes.

This complexity invites the multiscaled analysis to study the diversity of wave-transport scenarios even in such a simple systems as 1D Hertzian chains.

\section{Conclusion}

We have shown a diverse multimode character of wave transport scenarios in an inhomogeneous Hertzian chain at the intermittency described, from one side, by general discrete nonlinear governing equations and, from another side, by its asymptotic long wave approximations (the continuous limit). We obtain some new classes of solutions which correspond to particular conditions of their realization.

ISSN 2071-0194. Ukr. J. Phys. 2020. Vol. 65, No. 11
We show that the nonlinearity, size of the system, and ordering play significant roles in the selection of transport scenarios. Naturally, the linearization of the equations which govern the mechanical impulse transport in the inhomogeneous nonlinear 1D Hertzian force chain, did not bring us to a solitonic solution which is typical of that kind of problems [4].

We show analytically that, in the case of $1 \mathrm{D}$ inhomogeneous Hertz chain with weakly overlapped beads in the linear approximation, sharp-peaked modes can propagate, as well as cylindrical quasinormal modes. Sharp peaked solutions which have a pronounced asymmetry show a certain reminiscence with Fanotype resonances.

In [25], where the dynamics of Hertz chains has been studied, these modes are called RamsauerTownsend effect.

Singular modes has been also outlined recently after the numerical analysis [22] and interpreted to occur due to rare-wave-resonances.

We conclude that the initially weakly nonlinear inhomogeneous chain even in the linear approximation still shows some features which are more typical of the nonlinear behavior (in particular, in the form of Fano-type modes (resonances)).

Althoug, our consideration does not account for the dissipation effects, the obtained results could be practical, when these effect are negligibly small and contribute just through amplitudes of the considered wave modes $[20,21]$.

Therefore, under the appropriate values of the parameters (nonlinearity, inhomogeneity, signal amplitudes), the linearized inhomogeneous Hertzian chain allows both soliton-like and normal mode scenarios of the initial pulse transmission. In another words, the 1D Hertzian chain with Dyson hierarchy demonstrates a somewhat like drosseling property which is manipulated by a disorder with respect to a selected scenario of the wave transport.

In the perspective, the interplay between the nonlinearity, inhomogeneity, disorder, and dissipation and their influence on transporting modes dynamics will be a focus of our research.

We conclude that the multiscale approach can be an effective tool for studying the wave transport in anisotropic media at the intermittency between linear and nonlinear scenarios. 
The work was sponsored by the Ministry of Education and Science of Ukraine (grant No. 0116U008375).

1. E. Fermi, J. Pasta, S. Ulam, M. Tsingou. Studies of nonlinear problems. Los Alamos Sci. Lab. Rep. LA-1940, 978 (1955).

2. P.L. Bhatnagar. Nonlinear Waves in One-Dimensional Dispersive Systems (Clarendon, 1979) [ISBN: 9780198535317].

3. Y. Xu, V.F. Nesterenko. Propagation of short stress pulses in discrete strongly nonlinear tunable metamaterials. Phil. Trans. R. Soc. A 372, 20130186 (2014).

4. V. Nesterenko. Dynamics of Heterogeneous Materials (Springer, 2001) [ISBN: 978-1-4419-2926-6].

5. C. Coste, E. Falcon, S. Fauve. Solitary waves in a chain of beads under Hertz contact. Phys. Rev. E 56, 6104 (1997).

6. S. Sen, J. Hong, J. Bang, E. Avalos. R. Doney. Solitary waves in the granular chain. Phys. Rep. 462, 21 (2008).

7. E. Hascoët, H.J. Herrmann, V. Loreto. Shock propagation in a granular chain. Phys. Rev. E 59, 3202 (1999).

8. U. Harbola, A. Rosas, A.H. Romero, M. Esposito, K. Lindenberg. Pulse propagation in decorated granular chains: an analytical approach. Phys. Rev. E 80, 051302 (2009).

9. E. Somfai, J.-N. Roux, J.H. Snoeijer, M. van Hecke, W. van Saarloos. Elastic wave propagation in confined granular systems. Phys. Rev. E 72, 021301 (2005).

10. O.I. Gerasymov, N. Vandewalle, A.Ya. Spivak, N.N. Khudyntsev, G. Lumay, S. Dorbolo, O.A. Klymenkov. Stationary states in a $1 \mathrm{D}$ system of inelastic particles. Ukr. J. Phys. 53, 1128 (2008).

11. O.I. Gerasymov, N. Vandewalle. On the exact solutions of the problem of impulsive propagation in an inhomogeneous granular chain. Dopov. Nac. akad. nauk Ukr. 8, 67 (2012)

12. G. Lumay, S. Dorbolo, O. Gerasymov, N. Vandewalle. Experimental study of a vertical column of grains submitted to a series of impulses. Eur. Phys. J. E 36, 16 (2013).

13. L.D. Landau, L.P. Pitaevskii, A.M. Kosevich, E.M. Lifshitz. Theory of elasticity, 3rd ed. (ButterworthHeinemann, 1986) [ISBN: 978-0750626330].

14. H. Bateman. Some simple differential difference equations and the related functions. Bull. Amer. Math. Soc. 49, 494 (1943).

15. M. Abramowitz, I.A. Stegun. Handbook of Mathematical Functions with Formulas, Graphs, and Mathematical Tables (Dover, 1964) [ISBN: 0-486-61272-4].

16. E. Pinney. Ordinary difference-differential equations (University of California Press, 1958)
17. F.J. Dyson. The dynamics of a disordered linear chain. Phys. Rev. 92, 1331 (1953).

18. N.H. March, M. Parrinello. Collective Effects in Solids and Liquids (Adam Hilger, 1982) [ISBN: 978-0852745281].

19. A.D. Polyanin, V.F. Zaitsev. Handbook of Exact Solutions for Ordinary Differential Equations, 2nd ed. (Chapman and Hall/CRC, 2003) [ISBN: 978-1584882978].

20. A. Rosas, K. Lindenberg. Pulse dynamics in a chain of granules with friction. Phys. Rev. E 68, 041304 (2003).

21. R. Carretero-Gonzalez, D. Khatri, M.A. Porter, P.G. Kevrekidis, C. Daraio. Dissipative solitary waves in granular crystals. Phys. Rev. Lett. 102, 024102 (2009).

22. U. Fano. Effects of configuration interaction on intensities and phase shifts. Phys. Rev. 124, 18661961.

23. H. Yasuda, C. Chong, J. Yang, P.G. Kevrekidis. Emergence of dispersive shocks and rarefaction waves in power-law contact models. Phys. Rev. E 95, 062216 (2017).

24. O.I. Gerasymov. Physics of Granular Materials (TES, 2015) [ISBN: 978-617-7054-82-4].

25. A.J. Martinez, H. Yasuda, E. Kim, P.G. Kevrekidis, M.A. Porter, J. Yang. Scattering of waves by impurities in precompressed granular chains. Phys. Rev. E 93, 052224 (2016).

Received 02.12.19

О.І. Герасимов, А.Я. Співак

ЩОДО ХВИЛЬОВОГО ПЕРЕНОСУ

У СЛАБКО ЗБУРЕНОМУ ТА НЕОДНОРІДНОМУ

НЕЛІНІЙНОМУ СИЛОВОМУ ЛАНЦЮЖКУ

Р е $з$ ю м е

Отримано аналітичні та числові розв'язки рівнянь, які описують перенос механічного збурення в одновимірному попередньо слабко стиснутому герцівському ланцюжку. Досліджено системи як обмеженої, так і нескінченної довжини. Задача розв'язується як у дискретному, так і у континуальному формулюванні. Декілька сімейств аналітичних розв'язків у вигляді квазінормальних хвиль і характерних резонансних мод отримано в лінійному наближенні для слабко збурених неоднорідних систем. Знайдені резонансні моди пропонується тлумачити як резонанси типу Рамзауера-Таунсенда (або Фано), які з'являються завдяки неоднорідності. Отримані аналітичні результати порівнюються з числовими розв'язками дискретних рівнянь. Спостерігається сценарій різного масштабу переносу імпульсу збурення у неоднорідно навантаженому ланцюжку, який міг би реалізуватися або в асимптотиці, або між дискретною чи неперервною границями сформульованої задачі. Також проаналізовано роль розупорядкованості за допомогою моделі Дайсона. 